\title{
CARACTERÍSTICAS PALINOLÓGICAS DE LAS MIELES DE CASTAÑO DE GALICIA (NO ESPAÑA).
}

\author{
M. Carmen SEIJO, Victoria JATO, M. Isabel IGLESIAS y M. Jesús AIRA
}

\begin{abstract}
RESUMEN. Características palinológicas de las mieles de castaño de Galicia (NO España). Se han estudiado melisopalinológicamente 83 muestras de miel de Galicia con porcentajes superiores al $70 \%$ de polen de Castanea. El $46 \%$ de las muestras presenta porcentajes de este tipo polínico comprendidos entre el $70 \%$ y el $79 \%$, el $43 \%$ valores entre el $80 \%$ y el $89 \%$ y el $11 \%$ restante valores de este polen superiores al $89 \%$. El número medio de tipos polínicos es de 21 y el índice de mielada es nulo o muy bajo para todas las muestras. En cuanto a la riqueza polínica presentan una media de 26.384 granos de polen por gramo de miel. Otros tipos polínicos presentes en estas mieles son: Rubus, Erica, Eucalyptus, Cytisus y Campanula. Este tipo de mieles se producen, principalmente, en las provincias de Lugo y Ourense. En Lugo, la zona centro y las sierras orientales son las de mayor producción. En Ourense lo son las zonas de montaña de las sierras de Manzaneda, Queixa, San Mamede, el valle del Sil y el límite de la provincia con Portugal. En la provincia de Pontevedra la principal zona de producción es la comarca del Deza, mientras que en A Coruña es la zona Norte. Las principales características diferenciadoras de las mieles de castaño gallegas con respecto a otras de producción española son la ausencia de Lavandula stoechas, Cistus ladanifer, Clematis vitalba, Melilotus y la escasa presencia de Calluna vulgaris.
\end{abstract}

Palabras clave: Miel, Castanea, Galicia, melisopalinología, España.

ABSTRACT. Palynological characterization of sweet chestnut honeys from Galicia (NW Spain). We study 83 honey samples from Galicia, with percentages higher than $70 \%$ of Castanea pollen. The $46 \%$ of the samples show percentages of this pollen between $70 \%$ and $79 \%$, the $43 \%$ show values between $80 \%$ and $89 \%$ and the rest $(11 \%)$ values higher than $89 \%$. The mean of pollen types was 21 and the honeydew index was zero or very low for all the samples. Related to the pollen content, the samples have an average of 26.384 pollen grains per gramme of honey. Other pollen types appearing in these honey are: Rubus, Erica, Eucalyptus, Cytisus and Campanula. This honey type is produced, principally, in Lugo and Ourense provinces. In Lugo, the center area and the eastern mountains are the main important production localities. In Ourense, the honeys come from mountaineous areas of Manzaneda, Queixa, San Mamede, the Sil valley and the limit of the province with Portugal. In Pontevedra Province, the main important production area is the Deza area, while in A Coruña province its geographical origin is Northern. The main differences of the Galician chestnut honey with other Spanish honey of the same floral origin are the absence of Lavandula stoechas, Cistus ladanifer, Clematis vitalba, Melilotus and the scarce of Calluna vulgaris.

Keywords: Honey, Castanea, Galicia, melissopalynology, Spain.

\section{INTRODUCCIÓN}

Castanea sativa Miller es el taxón arbóreo de mayor interés apícola en el interior de
Galicia. Se encuentra en formaciones boscosas junto a Quercus robur L., en fragas monoespecíficas, o bien de forma aislada. Su abundancia es cada vez menor, debido 
principalmente a la deforestación, los incendios forestales y el abandono de la actividad agraria. Sin embargo, en las zonas de montaña menos alteradas permanecen todavía bosques de esta especie de considerable extensión.

Los cultivares tradicionales se distribuyen básicamente en las áreas de montaña de las provincias de Ourense y Lugo y en la comarca del Deza de la provincia de Pontevedra (Fernández \& Pereira, 1993).

Su período de floración comienza a mediados del mes de Junio permaneciendo hasta mediados de Julio. Debido a las variaciones climáticas, es posible encontrar en zonas relativamente cercanas una oscilación de su floración de 2-3 semanas. Es un recurso apícola excelente, pues proporciona abundante cantidad de néctar y polen (Bonet et al., 1985; Crane et al., 1984; Gómez, 1985), en una época en la que la colonia de abejas posee las condiciones biológicas ideales para una buena recolección. Aunque es una especie en la que la producción de mielatos puede complementar en buena medida su interés apícola, en Galicia es poco importante (Jato et al., 1991; Seijo, 1994; Meixide, 1987).

\section{MATERIAL Y MÉTODOS}

Se han estudiado desde el punto de vista melisopalinnlógico, 83 muestras de miel de Galicia que presentan más del $70 \%$ de polen de Castanea. En función de este porcentaje y de sus características organolépticas se han considerado monoflorales (Louveaux, 1968; Valencia, 1991; Jato et al., 1995; Iglesias et al., 1993).

Estas muestras se han seleccionado a partir de 530 mieles procedentes de toda Galicia que fueron sometidas a análisis melisopalinológico. La recogida fue realizada directamente a los apicultores, indicando para cada una de las muestras: la fecha de cosecha, la cantidad de miel recogida por el apicultor, el número de colmenas del colmenar y su situación geográfica.

La metodología utilizada es la propuesta por Louveaux et al. (1978). El análisis cualitativo se ha realizado sobre un mínimo de 1.200 granos de polen por muestra. El análisis cuantitativo y el Indice de mielada se ha realizado a partir de 10 gramos de miel. Como material de referencia para la identificación de los tipos polínicos se han utilizado preparaciones microscópicas de las plantas más abundantes en los alrededores de los colmenares.

Con el fin de realizar una mejor separación estadística de las muestras se ha utilizado una técnica multivariante cuya función consiste en agrupar los casos en subgrupos o conglomerados utilizando una medida de similaridad. Para facilitar el tratamiento estadístico se han seleccionado, en los espectros polínicos de las mieles, los taxones que representan valores superiores al $5 \%$ y que por este motivo tienen importancia en la composición polínica de las muestras estudiadas.

En la figura 1 se representan las zonas en las que se han recogido muestras que presentan más del $60 \%$ de polen de Castanea. En ellas es posible la producción de mieles con altos porcentajes de este taxón y en consecuencia monoflorales.

\section{RESULTADOS}

El número medio de tipos polínicos por muestra es 21 , con un máximo de 35 y un mínimo de 8.

Castanea presenta valores que oscilan entre el $70 \%$ y el $93 \%$. El $11 \%$ de las muestras presentan valores de polen de Castanea superiores al $90 \%$; el $43 \%$ valores entre $80 \%$ y el $89 \%$ y el $46 \%$ restante porcentajes comprendidos entre el $70 \%$ y el $79 \%$.

Entre los pólenes secundarios más 


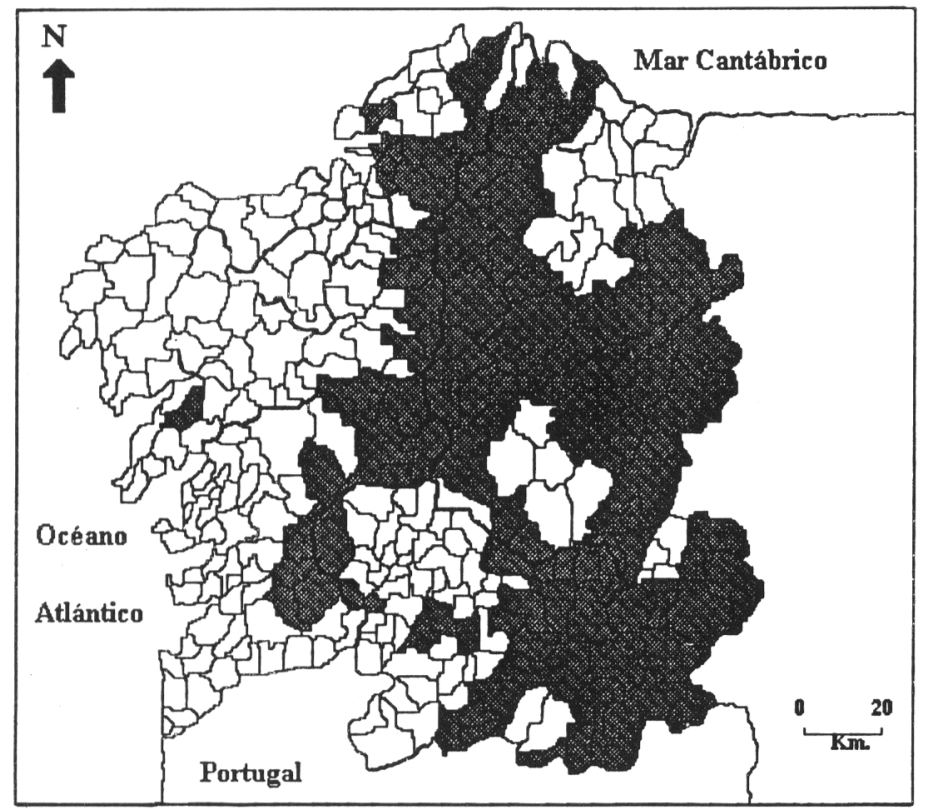

Figura 1. Zonas cuyas mieles presentan elevados porcentajes de polen de Castanea. Areas with the highest percentages of Castanea pollen in their honeys.

importantes (tab. 1) es de destacar el género Rubus que está presente en el $99 \%$ de las muestras, siendo su máximo valor $24 \%$. Erica es otro género muy común en estas mieles, estando presente en el $95 \%$ con un máximo de $15 \%$. Los tipos polínicos Cytisus y Campanula son también, muy frecuentes $(90 \%$ y $77 \%$ de las muestras, respectivamente) y su máximo es de $13 \%$ y $11 \%$. Trifolium y Quercus se han identificado en el $75 \%$ y $71 \%$ de las muestras siendo sus respectivos valores máximos de $5 \%$ y $2 \%$. Otros tipos polínicos (Eucalyptus, Echium, Lotus, Centaurea y Scrophularia), aunque frecuentes, están presentes en menos del $70 \%$ de las mieles siendo sus máximos valores inferiores al 10\%, a excepción de Eucalyptus (17\%).

En la figura 2 se representa un espectro polínico tipo para las mieles de castaño de Galicia. Para ello se han elegido los valores medios de cada uno de los taxones de importancia en las muestras de miel analizadas, al mismo tiempo se representan los valores máximos y mínimos con el fin de acotar el rango de aparición de estos tipos polínicos.

En cuanto al análisis cuantitativo, 35 muestras pertenecen a la clase III de Maurizio, 31 a la clase II, 10 a la clase IV, 5 a la clase I y las 2 restantes a la clase V. La media de granos de polen por gramo de miel es de 26.384. No se han encontrado diferencias significativas entre el contenido en polen de Castanea en las mieles y su riqueza polínica (granos de polen por gramo de miel) (tab. 2).

La presencia de elementos indicadores de mielada fue nula o muy baja $(\mathrm{HDE} / \mathrm{P}<0.1)$ en todas las muestras.

El análisis estadístico ha permitido obtener un gráfico en el que se diferencian 7 grupos de muestras cada uno de ellos con características propias (fig. 3). El primero está formado exclusivamente por una muestra que presenta un $13 \%$ del tipo polínico Cytisus, y por ello es netamente distinta a las demás. El 


\begin{tabular}{|c|c|c|c|c|c|c|c|c|c|}
\hline FAMILIA & TIPO POLÍNICO & D & A & I & $\mathrm{R}$ & $P$ & N.P. & $\%$ & V. A. \\
\hline ACERACEAE & Acer & - & - & - & - & 1 & 82 & 1 & $\mathrm{P}, \mathrm{M}$ \\
\hline AQUIFOLIACEAE & Ilex aquifolium & - & - & - & - & 7 & 76 & 8 & $\mathrm{~N}, \mathrm{P}$ \\
\hline ARALIACEAE & Hedera helix & - & - & - & 3 & 5 & 75 & 10 & $\mathrm{~N}, \mathrm{P}$ \\
\hline \multirow[t]{2}{*}{ BETULACEAE } & Alnus glutinosa & - & - & - & - & 4 & 79 & 5 & $\mathrm{P}, \mathrm{M}$ \\
\hline & Betula alba & - & - & - & - & 6 & 77 & 7 & $\mathrm{P}, \mathrm{M}$ \\
\hline \multirow[t]{4}{*}{ BORAGINACEAE } & Anchusa & - & - & - & - & 8 & 75 & 10 & $\mathrm{~N}, \mathrm{P}$ \\
\hline & Echium & - & - & - & 4 & 44 & 35 & 58 & $\mathrm{~N}, \mathrm{P}$ \\
\hline & Lithodora & - & - & - & - & 13 & 70 & 16 & $\mathrm{~N}, \mathrm{P}$ \\
\hline & Myosotis & - & - & - & - & 16 & 67 & 19 & $\mathrm{~N}, \mathrm{P}$ \\
\hline CAMPANULACEAE & t. Campanula & - & - & 8 & 10 & 46 & 19 & 77 & $\mathrm{~N}, \mathrm{P}$ \\
\hline \multirow[t]{2}{*}{ CAPRIFOLIACEAE } & Lonicera & - & - & - & - & 1 & 82 & 1 & $\mathrm{~N}, \mathrm{P}$ \\
\hline & Viburnum & - & - & - & - & 3 & 80 & 4 & $\mathrm{~N}, \mathrm{P}$ \\
\hline \multirow[t]{3}{*}{ CARYOPHYLLACEAE } & Corrigiola & - & - & - & - & 1 & 82 & 1 & $\mathrm{P}$ \\
\hline & t. Silene & - & - & - & - & 6 & 77 & 7 & $\mathrm{~N}, \mathrm{P}$ \\
\hline & t. Stellaria & - & - & - & - & 1 & 82 & 1 & $\mathrm{~N}, \mathrm{P}$ \\
\hline \multirow[t]{6}{*}{ CISTACEAE } & t. Cistus ladanifer & - & - & - & - & 8 & 75 & 10 & $\mathrm{P}$ \\
\hline & Cistus psilosepalus & - & - & - & - & 24 & 59 & 29 & $\mathrm{P}$ \\
\hline & Cistus salvifolius & - & - & - & - & 10 & 73 & 12 & $\mathrm{P}$ \\
\hline & Cistus sp. & - & - & - & & 10 & 73 & 12 & $\mathrm{P}$ \\
\hline & t. Halimium & - & - & - & 1 & 4 & 78 & 6 & $\mathrm{P}$ \\
\hline & Helianthemum & - & - & - & - & 11 & 72 & 13 & $\mathrm{P}$ \\
\hline \multirow[t]{7}{*}{ COMPOSITAE } & t. Anthemis & - & - & - & - & 17 & 66 & 20 & $\mathrm{~N}, \mathrm{P}$ \\
\hline & t. Bellis & - & - & - & - & 4 & 79 & 5 & $\mathrm{~N}, \mathrm{P}$ \\
\hline & Centaurea & - & - & - & 1 & 40 & 42 & 49 & $\mathrm{~N}, \mathrm{P}$ \\
\hline & Cirsium & - & - & - & - & 2 & 81 & 2 & $\mathrm{~N}, \mathrm{P}$ \\
\hline & t. Helianthus апnииs & - & - & - & - & 1 & 82 & 1 & $\mathrm{~N}, \mathrm{P}$ \\
\hline & t. Senecio & - & - & - & - & 14 & 69 & 17 & $\mathrm{~N}, \mathrm{P}$ \\
\hline & t. Taraxacum oficcinale & - & - & - & - & 37 & 46 & 45 & $\mathrm{~N}, \mathrm{P}$ \\
\hline CRASSULACEAE & Sedum & - & & - & - & 17 & 66 & 20 & $\mathrm{~N}, \mathrm{P}$ \\
\hline \multirow[t]{3}{*}{ CRUCIFERAE } & t. Brassica & - & - & - & 2 & 36 & 45 & 46 & $\mathrm{~N}, \mathrm{P}$ \\
\hline & t. Capsella bursapastoris & - & - & - & - & 5 & 78 & 6 & $\mathrm{~N}, \mathrm{P}$ \\
\hline & t. Raphanus & - & - & 1 & - & 15 & 67 & 19 & $\mathrm{~N}, \mathrm{P}$ \\
\hline CUCURBITACEAE & t. Bryonia cretica & - & - & - & - & 2 & 81 & 2 & $\mathrm{~N}, \mathrm{P}$ \\
\hline \multirow[t]{7}{*}{ ERICACEAE } & Calluna vulgaris & - & - & - & 1 & 24 & 58 & 30 & $\mathrm{~N}, \mathrm{P}$ \\
\hline & Daboecia cantabrica & - & - & 1 & 2 & 11 & 69 & 17 & $\mathrm{~N}, \mathrm{P}$ \\
\hline & Erica arborea & - & - & 10 & 12 & 28 & 33 & 60 & $\mathrm{~N}, \mathrm{P}$ \\
\hline & Erica australis & - & - & 5 & 20 & 43 & 15 & 82 & $\mathrm{~N}, \mathrm{P}$ \\
\hline & t. Erica cinerea & - & - & 1 & 5 & 30 & 47 & 43 & $\mathrm{~N}, \mathrm{P}$ \\
\hline & Erica sp. & - & - & - & 2 & 23 & 58 & 30 & $\mathrm{~N}, \mathrm{P}$ \\
\hline & Erica umbellata & - & - & 3 & 6 & 33 & 41 & 51 & $\mathrm{~N}, \mathrm{P}$ \\
\hline EUPHORBIACEAE & Mercurialis & - & - & - & - & 5 & 78 & 6 & $\mathrm{~N}, \mathrm{P}$ \\
\hline \multirow[t]{2}{*}{ FAGACEAE } & Castanea sativa & 83 & - & - & - & - & - & 100 & $\mathrm{~N}, \mathrm{P}, \mathrm{M}$ \\
\hline & Quercus & - & - & 1 & 4 & 54 & 24 & 71 & P.M \\
\hline HYPERICACEAE & Hypericum & - & - & - & 1 & 9 & 73 & 12 & $\mathrm{~N}, \mathrm{P}$ \\
\hline JUGLANDACEAE & Juglans regia & - & - & - & - & 1 & 82 & 1 & $\mathrm{P}, \mathrm{M}$ \\
\hline \multirow[t]{6}{*}{ LABIATAE } & Ajuga & - & - & & - & 5 & 78 & 6 & $\mathrm{~N}, \mathrm{P}$ \\
\hline & t. Lamium & - & - & - & - & 2 & 81 & 2 & $\mathrm{~N}, \mathrm{P}$ \\
\hline & Lavandula stoechas & - & - & - & - & 6 & 77 & 7 & $\mathrm{~N}, \mathrm{P}$ \\
\hline & t. Mentha & - & - & - & - & 21 & 62 & 25 & $\mathrm{~N}, \mathrm{P}$ \\
\hline & t. Rosmarinus officinalis & - & - & - & - & 7 & 76 & 8 & $\mathrm{~N}, \mathrm{P}$ \\
\hline & Teucrium scorodonia & - & - & - & - & 13 & 70 & 16 & $\mathrm{~N}, \mathrm{P}$ \\
\hline \multirow[t]{4}{*}{ LEGUMINOSAE } & t. Cytisus & - & & 12 & 14 & 49 & 8 & 90 & $\mathrm{~N}, \mathrm{P}$ \\
\hline & t. Lotus & - & - & 6 & 6 & 35 & 36 & 57 & $\mathrm{~N}, \mathrm{P}$ \\
\hline & t. Trifolium & - & - & 5 & 6 & 51 & 21 & 75 & $\mathrm{~N}, \mathrm{P}$ \\
\hline & t. Vicia & - & - & - & - & 4 & 79 & 5 & $\mathrm{~N}, \mathrm{P}$ \\
\hline \multirow[t]{3}{*}{ LILIACEAE } & Allium & - & - & - & - & 5 & 78 & 6 & $\mathrm{~N}, \mathrm{P}$ \\
\hline & Asphodelus & - & - & - & - & 2 & 81 & 2 & $\mathrm{~N}, \mathrm{P}$ \\
\hline & Shimetis planifolia & - & - & - & - & 2 & 81 & 2 & $\mathrm{~N}, \mathrm{P}$ \\
\hline LYTHRACEAE & Lythrum & - & - & & - & 2 & 81 & 2 & $\mathrm{~N}, \mathrm{P}$ \\
\hline MALVACEAE & Malva & - & - & - & - & 3 & 80 & 4 & $\mathrm{~N}, \mathrm{P}$ \\
\hline MYRTACEAE & Eucalyptus & - & 2 & 8 & 7 & 32 & 34 & 59 & $\mathrm{~N}, \mathrm{P}$ \\
\hline
\end{tabular}




\begin{tabular}{|c|c|c|c|c|c|c|c|c|c|}
\hline FAMILIA & TIPO POLÍNICO & D & A & I & $\mathrm{R}$ & $\mathrm{P}$ & N.P. & $\%$ & V. A. \\
\hline \multirow[t]{3}{*}{ OLEACEAE } & t. Fraxinus & - & - & - & - & 2 & 81 & 2 & $\mathrm{P}, \mathrm{M}$ \\
\hline & t. Ligustrum & - & - & - & - & 13 & 70 & 16 & $\mathrm{~N}, \mathrm{P}$ \\
\hline & Olea europaea & - & - & - & - & 2 & 81 & 2 & $\mathrm{P}$ \\
\hline OXALIDACEAE & Oxalis & - & - & - & - & 1 & 82 & 1 & $\mathrm{~N}, \mathrm{P}$ \\
\hline PINACEAE & Pinus & - & - & - & - & 7 & 76 & 8 & $\mathrm{P}, \mathrm{M}$ \\
\hline PLANTAGINACEAE & Plantago & - & - & - & 1 & 39 & 43 & 48 & $\mathrm{P}$ \\
\hline \multirow[t]{2}{*}{ POACEAE } & Otras Poaceae & - & - & - & 1 & 36 & 46 & 45 & $\mathrm{P}$ \\
\hline & Zea mays & - & - & - & - & 14 & 69 & 17 & $\mathrm{P}$ \\
\hline \multirow[t]{2}{*}{ POLYGONACEAE } & Polygonum & - & - & - & - & 1 & 82 & 1 & $\mathrm{~N}, \mathrm{P}$ \\
\hline & Rumex & - & - & - & - & 11 & 72 & 13 & $\mathrm{~N}, \mathrm{P}$ \\
\hline PRIMULACEAE & Anagallis & - & - & - & - & 4 & 79 & 5 & $\mathrm{~N}, \mathrm{P}$ \\
\hline RANUNCULACEAE & Ranunculus & - & - & - & - & 9 & 74 & 11 & $\mathrm{~N}, \mathrm{P}$ \\
\hline \multirow[t]{2}{*}{ RESEDACEAE } & Reseda & - & - & - & - & 8 & 75 & 10 & $\mathrm{~N}, \mathrm{P}$ \\
\hline & Sesamoides & - & - & - & 1 & 9 & 73 & 12 & $\mathrm{~N}, \mathrm{P}$ \\
\hline RHAMNACEAE & Frangula alnus & - & - & - & - & 15 & 68 & 18 & $\mathrm{~N}, \mathrm{P}$ \\
\hline \multirow[t]{4}{*}{ ROSACEAE } & t. Crataegus monogyna & - & - & - & - & 30 & 53 & 36 & $\mathrm{~N}, \mathrm{P}, \mathrm{J}$ \\
\hline & Filipendula & - & - & - & - & 4 & 79 & 5 & $\mathrm{~N}, \mathrm{P}$ \\
\hline & t. Prunus & - & - & 4 & 5 & 24 & 50 & 40 & $\mathrm{~N}, \mathrm{P}, \mathrm{J}$ \\
\hline & t. Rubus & - & 15 & 61 & 4 & 2 & 1 & 99 & $\mathrm{~N}, \mathrm{P}, \mathrm{J}$ \\
\hline SALICACEAE & Salix & - & - & - & 1 & 30 & 52 & 37 & $\mathrm{~N}, \mathrm{P}$ \\
\hline SAXIFRAGACEAE & Saxifraga & - & - & - & - & 6 & 77 & 7 & $\mathrm{~N}, \mathrm{P}$ \\
\hline \multirow{4}{*}{ SCROPHULARIACEAE } & Anarrhinum & - & - & - & 3 & 18 & 62 & 25 & $\mathrm{~N}, \mathrm{P}$ \\
\hline & Digitalis purpurea & - & - & - & - & 5 & 78 & 6 & $\mathrm{~N}, \mathrm{P}$ \\
\hline & t. Scrophularia & - & - & - & - & 26 & 57 & 31 & $\mathrm{~N}, \mathrm{P}$ \\
\hline & Verbascum & - & - & - & - & 4 & 79 & 5 & $\mathrm{~N}, \mathrm{P}$ \\
\hline SOLANACEAE & Solanum & - & - & - & - & 5 & 78 & 6 & $\mathrm{~N}, \mathrm{P}$ \\
\hline ULMACEAE & Ulmus minor & - & - & - & - & 2 & 81 & 2 & $\mathrm{P}, \mathrm{M}$ \\
\hline \multirow[t]{4}{*}{ UMBELLIFERAE } & t. Conium maculatum & - & - & - & - & 11 & 72 & 13 & $\mathrm{~N}, \mathrm{P}$ \\
\hline & t. Daucus carota & - & - & - & - & 13 & 70 & 16 & $\mathrm{~N}, \mathrm{P}$ \\
\hline & t. Oenanthe croccata & - & - & - & - & 1 & 82 & 1 & $\mathrm{~N}, \mathrm{P}$ \\
\hline & t. Smyrnium olusatrum & - & - & - & - & 4 & 79 & 5 & $\mathrm{~N}, \mathrm{P}$ \\
\hline URTICACEAE & t. Urtica & - & - & - & - & 3 & 80 & 4 & $\mathrm{~N}, \mathrm{P}$ \\
\hline VIOLACEAE & Viola & - & - & - & - & 2 & 81 & 2 & $\mathrm{~N}, \mathrm{P}$ \\
\hline \multirow[t]{2}{*}{ VITACEAE } & Vitis vinifera & - & - & - & - & 1 & 82 & 1 & $\mathrm{~N}, \mathrm{P}, \mathrm{J}$ \\
\hline & Otros & - & - & - & - & 14 & 69 & 17 & - \\
\hline
\end{tabular}

Tabla 1. Clases de frecuencia de los tipos polínicos de las mieles de castaño. $D=\geq 45 \% ; A=15 \%-<45 \%$; $\mathrm{I}=3 \%-<15 \% ; \mathrm{R}=1 \%-<3 \% ; \mathrm{P}=<1 \% ; \mathrm{N} . \mathrm{P} .=$ Ausencia. V. A. = Valor Apícola; N: nectar, P: polen, M: mielato, J: jugos de frutas. \%= Presencia del tipo polínico en el total de las muestras. Frequency classes of the pollen types of sweet chestnut honeys. $D=\geq 45 \% ; A=15 \%-<45 \% ; I=3 \%-<15 \% ; R=1 \%-<3 \% ; P=$ $<1 \% ;$ N.P. = Absent. V. A. = Apicultural value; N: nectar, P: pollen, M: honeydew, J: Fruit juices \%= Presence of the pollen type in all honeys.

segundo grupo (5 muestras) se corresponde con mieles que presentan como segundo polen en importancia el de Eucalyptus. El tercero contiene 25 muestras con porcentajes muy elevados de Castanea (>80\%) y sin predominio de ningún tipo polínico secundario. El cuarto grupo (5 mieles) presenta porcentajes importantes de polen del tipo polínico Campanula. El quinto y el séptimo (22 y 15 muestras, respectivamente) son muy similares pero se diferencian en que el primero de ellos contiene mieles con porcentajes altos de
Castanea $(<80 \%)$ y Rubus como polen importante $(>10 \%)$ y el último presenta porcentajes de Castanea entre 70 y $80 \%$ y Rubus con porcentajes inferiores al $10 \%$. El sex to grupo (10 mieles) presenta como segundo polen en importancia el de Erica.

\section{DISCUSIÓN}

Entre las mieles monoflorales de Galicia, las de castaño son las de mayor producción 


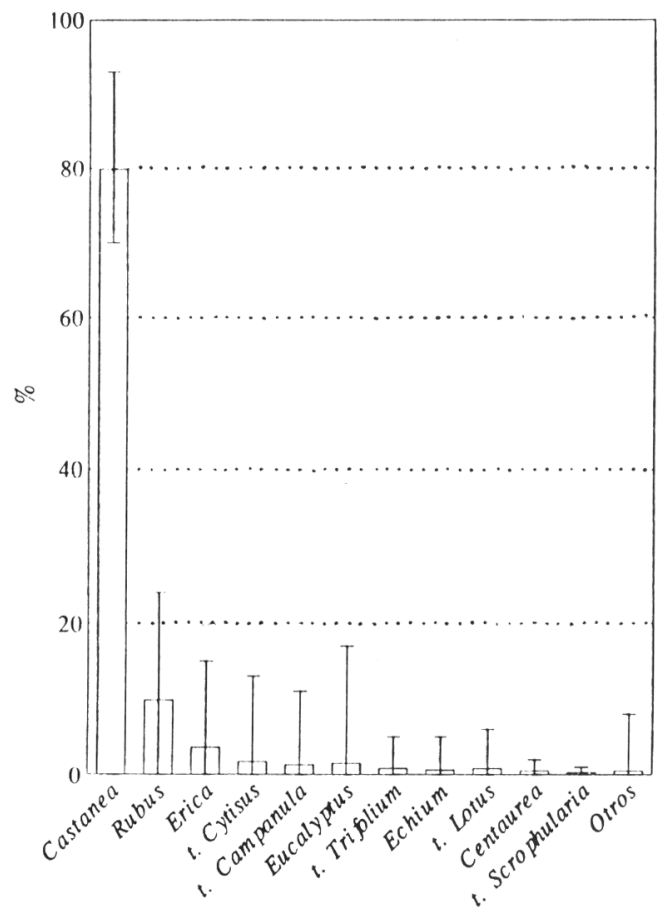

Figura 2. Valor medio, máximo y mínimo de cada uno de los tipos polínicos más importantes en las mieles de castaño de Galicia. Mean, maximum and minimum value of the most important pollen types of sweet chestnut honeys from Galicia.

(Seijo, 1994). La mayor parte proceden de la provincia de Lugo, principalmente de la zona centro (Lugo, Fonsagrada y A Ulloa) y de las sierras orientales (Ancares y El Caurel). En la provincia de Orense estas mieles se producen en las comarcas de las sierras de Manzaneda, Queixa, San Mamede, Invernadeiro, en el Valle del Sil y en el límite de la provincia con Portugal. En la provincia de Pontevedra, se producen en la comarca del Deza, mientras que en A Coruña, el lugar más adecuado es el Norte (fig. 1). Estas zonas de producción coinciden, mayoritariamente, con las zonas de distribución de castañares cultivados tradicionalmente en Galicia (Fernández \& Pereira, 1993), a excepción de algunas zonas del centro de la provincia de Ourense, en donde el abandono de la actividad agrícola ha provocado extensiones muy importantes del género Rubus que condicionan fuertemente el tipo de miel producida en los colmenares. A pesar de la existencia de distintas zonas de producción dentro del territorio gallego no se han encontrado diferencias significativas entre las mieles de castaño de distinta procedencia geográfica.

El estudio polínico de las mieles de Galicia con porcentajes superiores al $70 \%$ de Castanea ha demostrado que el taxón acompañante de mayor importancia en estas mieles es Rubus. Este género presenta un período de floración de mayor duración que Castanea sativa y que, en su inicio, se solapa con él. Una vez acabada la floración de Castanea sativa las abejas siguen recogiendo néctar en Rubus. Este néctar pasa directamente a las alzas almacén de las colmenas diluyendo en cierto modo el néctar recogido de Castanea sativa. Si la floración de Rubus es muy abundante se originan masivamente mieles milflorales cuyo origen floral son ambos taxones: Castanea sativa y Rubus. Por ese motivo, aunque en Galicia Castanea sativa está presente prácticamente en todo el territorio, sólo en aquellas zonas dónde es muy abundante es posible recolectar este tipo de miel. Algunos apicultores cosechan esta miel monofloral extrayendo los panales de las colmenas al final de la floración de Castanea sativa, no permitiendo la entrada masiva de néctar de Rubus ya que, en principio los aportes melíferos del castaño son más atractivos para las abejas que Rubus.

Como se puede observar en el espectro polínico tipo los demás taxones secundarios presentan, en general, poca importancia en las mieles de este origen (siempre con valores inferiores al 2\%) a excepción de Erica (E. australis, E. umbellata, E. arborea y tipo polínico $E$. cinerea) que es muy abundante en el matorral gallego y en su conjunto florece desde principios del mes de Marzo hasta septiembre.

En cuanto a la cantidad de granos de polen 


\begin{tabular}{cccc}
\hline $\begin{array}{c}\text { Clase de } \\
\text { Maurizio }\end{array}$ & $\begin{array}{c}\text { Rango (granos de } \\
\text { polen/gr. miel) }\end{array}$ & $\begin{array}{c}\text { Rango (\% de } \\
\text { Castanea) }\end{array}$ & $\begin{array}{c}\mathbf{N}^{\text {N de }} \\
\text { mieles }\end{array}$ \\
\hline I & $489-603$ & $70-72$ & 2 \\
II & $2.476-9.441$ & $70-91$ & 31 \\
III & $10.150-48.860$ & $71-93$ & 35 \\
IV & $51.001-71.501$ & $70-91$ & 10 \\
V & $108.627-188.400$ & $71-89$ & 5 \\
\hline
\end{tabular}

Tabla 2. Clasificación de Maurizio y contenido en polen de Castanea. Maurizio's classification and Castanea pollen content.

por gramo de miel, son mieles con una riqueza polínica media, aunque el 18\% de los casos presentan cantidades de polen incluídas en las clases IV y V de Maurizio. El 40\% de las muestras presentan valores inferiores a 10.000 granos de polen por gramo de miel. Esto puede ser debido a que son mieles extraídas por centrifugación y proceden de colmenas de alzas de desarrollo vertical.

Aunque Castanea sativa y Quercus, son taxones sobre los que con frecuencia se producen mielatos (Crane \& Walker, 1985), en las mieles estudiadas es escasa la presencia de elementos indicadores de mielada. Estas

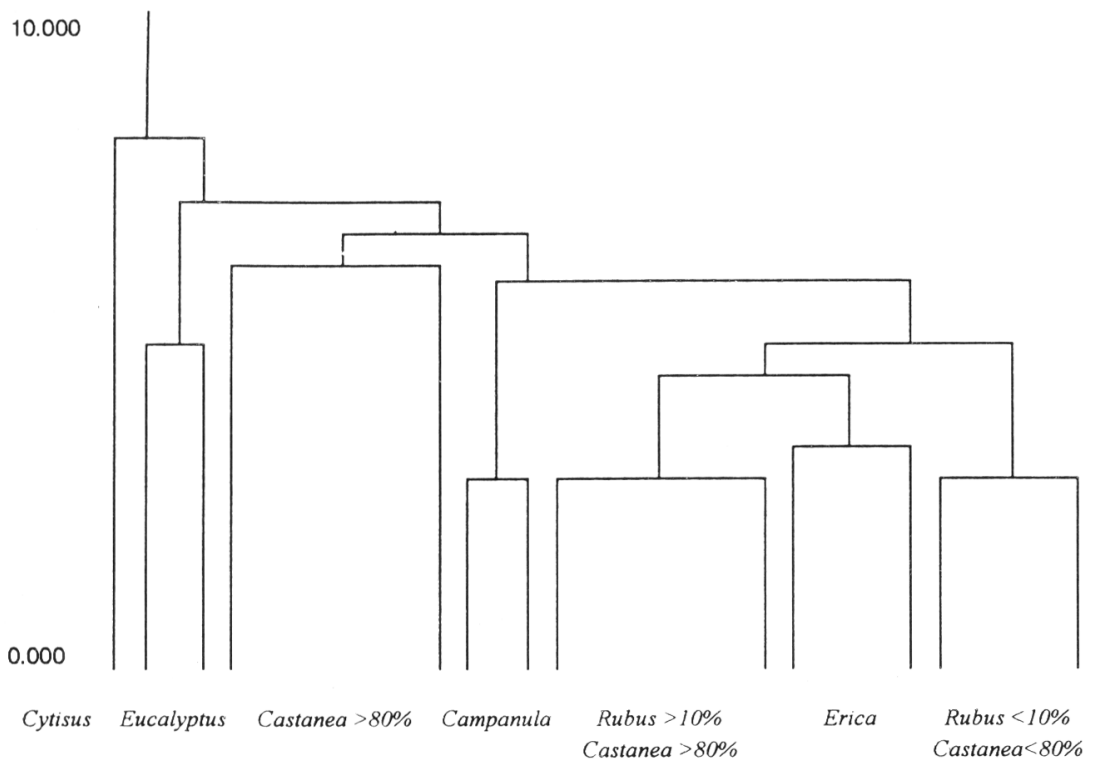

Figura 3. Dendrograma de agrupamiento de las mieles estudiadas. Cluster of the honeys studied. 
pseudacacia L., Erica vagans L. y Clematis Vitalba las diferencia de las gallegas. En las mieles de Zamora (Gómez, 1989) los pólenes acompañantes son: Calluna vulgaris, Erica sp., Halimium sp., Lavandula stoechas y Genista sp.. La escasez $(<0,1 \%)$, en las de Galicia, de Lavandula stoechas y la baja presencia de Halimium y Calluna vulgaris las diferencia.

En los Alpes se producen mieles de este origen floral, pero la elevada presencia de polen de Myosotis y Fraxinus caracteriza a estas mieles (Vorwohl, 1972). Otras zonas de producción son Francia, Italia, Yugoslavia y Turquía (Ricciardelli \& Vorwohl, 1979). El polen de Tilia e importantes cantidades de polen de Trifolium diferencia a las mieles francesas, de este origen floral, de las gallegas. De las mieles italianas se diferencian por la ausencia de Citrus, Melilotus, Olea y Tordylium. La presencia en las mieles turcas de pólenes de Rhus, Cucumis, Citrullus y Erica manipuliflora L. constituye una buena característica diferenciadora.

\section{CONCLUSIÓN}

A modo de conclusión, las mieles de castaño son las más importantes en cuanto a la producción de mieles monoflorales en Galicia (de las mieles monoflorales estudiadas el $39 \%$ son de castaño, el $28 \%$ de eucalipto, el $23 \%$ de zarza, el $6 \%$ de brezo y el $4 \%$ restante de diversas Leguminosae). Se caracterizan por presentar porcentajes importantes de polen de Rubus, Erica, t. Campanula, t. Cytisus, Trifolium, Quercus y, en menor medida, Echium y Eucalyptus. Destacando la baja presencia de pólenes de Cistaceae, Helianthus annuus, Olea europaea, Lavandula stoechas, etc... frecuentes en otras mieles de este origen floral.

\section{BIBLIOGRAFÍA}

BONET, A., J. RITA y M.T. SEBASTIA - 1985- La flora melifera de la circumscripció de Barcelona. Diputació de Barcelona. 107 pp.
CRANE, E., P. WALKER \& R. DAY -1984Directory of important world honey sources. International Bee Research Association. 384 pp.

CRANE, E. \& P. WALKER - 1985- Important honeydew sources and their honeys. Bee World 3: 105-112.

FERNÁNDEZ, J. y S. PEREIRA -1993-Inventario y distribución de los cultivares tradicionales de castaño (Castanea sativa Mill.) en Galicia. M.A.P.A.

GÓMEZ FERRERAS, C. -1985-Estudio polínico de mieles españolas. Tesis Doctoral. Universidad Complutense de Madrid.

GÓMEZ FERRERAS, C. -1989- Contribución al análisis polínico de mieles de la provincia de Zamora. Botanica Complutensis 14: 157-165.

IGLESIAS, M.I., M.V. JATO y M.J. AIRA -1993Estudio palinológico de mieles de montaña de la provincia de Ourense (NW España). Acta Bot. Malacitana 18: 119-123.

JATO M.V., A. SALA-LLINARES, I. IGLESIAS \& M. SUÁREZ-CERVERA -1991- Pollens of honeys from Northwestern Spain. Journal of Apicultural Research 30 (2):69-73.

JATO, M.V., M.I. IGLESIAS, J. GARCÍA, M. MARSÁ \& M.C. SEIJO -1995- Caratteristiche melissopalinologiche e fisico-chimiche dei mieli di Rubus e di Castanea della provincia de Orense (N.O. Spagna). Apicoltura 10: 11-22

LOUVEAUX, J. -1968- L'analyse pollinique des miels (in Chauvin, R. Les produits de la ruche). Masson et Cie. Paris. T3:325-362.

LOUVEAUX, J. A. MAURIZIO \& G. VORWOHL -1978- Methods of Melissopalynology. Bee World 59 (4):139-157.

LUIS VILLOTA DE, P. y C. GÓMEZ FERRERAS -1989- Contribución al análisis polínico de mieles de Asturias Occidental (España). Botanica Complutensis 15:163-173

MEIXIDE VILARIÑN, J.L. -1987- Contribución al estudio de los áfidos productores de mielato en Galicia. Su aprovechamiento por la abeja melífera. Boletín de la A.G.A. 24:25-32.

PÉREZ DE ZABALZA MADOZ, A. - 1989-Estudio 
palinológico de las mieles de Navarra. Tesis Doctoral. Universidad de Navarra.

RICCIARDELLI D'ALBORE, G.C. \& G. VORWOHL - 1979- Tipos de miel monoflora de la región del Mediterráneo verificados mediante análisis microscópicos de la miel. XXVII Cong. Internacional de Apicultura. 201-208.

SEIJO COELLO, M.C. -1994- Caracterización de la miel de Galicia a través del espectro polínico. Tesis Doctoral. Universidad de Vigo.

VALENCIA BARRERA, R. -1991-Estudio palinológico de mieles de la Provincia de León. Tesis Doctoral. Universidad de León.
VORWOHL, G. -1972- Das pollenspektrum von honigen aus den italienischen alpen. Apidologie 3(4):309-340

Aceptado para su publicación en Junio de 1997

Dirección de los autores. M.C. SEIJO, V. JATO y M.I. IGLESIAS: Dpto. de Recursos Naturales y Medio Ambiente. Facultad de Ciencias. Edificio Politécnico. Universidad de Vigo. Campus «As Lagoas». 32004 Orense. España. M.J. AIRA: Dpto. Biología Vegetal. Facultad de Farmacia. Campus Sur. Universidad de Santiago de Compostela. 15706 Santiago de Compostela. España. 